LBL-35789

\title{
The Use of Transgenic Animals to Study Lipoprotein Metabolism
}

\author{
Edward M. Rubin and Andrew S. Plump
}

Life Sciences Division

Lawrence Berkeley Laboratory

University of California

Berkeley, California 94720

December 1993

\section{DISCLAIMER}

\begin{abstract}
This report was prepared as an account of work sponsored by an agency of the United States Government. Neither the United States Government nor any agency thereof, nor any of their employees, makes any warranty, express or implied, or assumes any legal liability or responsibility for the accuracy, completeness, or usefulness of any information, apparatus, product, or process disclosed, or represents that its use would not infringe privately owned rights. Reference herein to any specific commercial product, process, or service by trade name, trademark, manufacturer, or otherwise does not necessarily constitute or imply its endorsement, recommendation, or favoring by the United States Government or any agency thereof. The views and opinions of authors expressed herein do not necessarily state or reflect those of the United States Government or any agency thereof.
\end{abstract}

This work was supported by National Institutes of Health Grant PPG HL-18574, National Dairy Promotion and Research Board through the U.S. Department of Energy under Contract No. DE-AC03-76SF00098. 


\section{DISCLAIMER}

Portions of this document may be illegible in electronic image products. Images are produced from the best available original document. 
Transgenics: chapter

Authors: Edward M. Rubin, M. D., Ph. D.; Andrew S. Plump, Ph. D.

Text font: Times (12 pt.)

\section{Introduction}

The application of transgenic technology to lipoprotein metabolism and atherosclerosis has been a rapidly emerging field. The first lipoprotein related transgenes were reported in 1988. Today, a large percentage of the genes involved in lipoprotein metabolism have been overexpressed in mice, and a substantial number of these same genes have been disrupted by homologous recombination in embryonic stem (ES) cells. The utility of animal models of lipoprotein metabolism and atherosclerosis is far-reaching given the complex nature of these systems. There are at least 17 known genes directly involved in lipoprotein metabolism (and in all likelihood dozens more that are directly or indirectly involved) (reviewed in Breslow, 1988). This massive network of interacting factors has necessitated the development of in vivo systems which can be subject to genetic manipulation.

Transgenic technology allows the overexpression of any gene in the mouse. The power of overexpression is obvious: elucidating function in a relatively controlled genetic environment in which the whole system is present and operational. The not-so-obvious problem with transgenics is "background," or for purposes of the current discussion, the mouse's own lipoprotein system. While many elements of the human and murine lipoprotein systems are similar, enough differences exist to complicate the interpretation of most transgenic models. With the advent of gene knockout, we have been given the ability to overcome "background." By recreating the genetic complement of the mouse we can alter a system in essentially any manner desired. As unique tools, and in combination with one another, the overexpression of foreign genes and the targeted disruption or alteration of endogenous genes has already and will continue to offer a wealth of information on the biology of lipoprotein metabolism and its effect on atherosclerosis susceptibility. 
Introduction into transgenic and gene knockout technology. When thinking about the mouse as a biological system, one must consider three technical capabilities: gene mapping and positional cloning, transgene overexpression, and gene knockout. Gene mapping and positional cloning are the most arduous and time consuming of the techniques. An improved genetic and physical map has vastly improved an investigator's ability to identify quantitative trait loci that can influence most measurable phenotypes. The two other techniques are more directly relevant to this chapter and are techniques that have been actively refined over the past decade. Though at times technically demanding, both are well-implementable and reproducible in many laboratories.

As seen in Figure 1, overexpression involves the microinjection of multiple copies of a foreign piece of DNA into the pronucleus of a 12 hour fertilized mouse embryo. The piece of DNA can be any size, derived from a plasmid, a cosmid, a phagemid, or a yeast artificial chromosome. It can be from any species. Through a poorly understood set of events the foreign gene can integrate into the genome of the fertilized egg. The number of copies of the gene which integrate (multiple copies of genes frequently arise by concatermerization prior to integration) and the site of integration are random. Both appear to eventually influence levels of expression. After injection of the fertilized egg, it is surgically reimplanted into the oviduct of a pseudopregnant surrogate mother which carries the embryo to term. At weaning a Southem blot or PCR analysis of DNA extracted from the tail tip can help to identify mice in which an integration event has occurred. RNA and protein analysis are used to evaluate which of the founder animals are expressing the integrated gene and at what levels (Hogan, et al., 1986).

Gene knockout is a more time consuming process. It is a technique rooted in years of pioneering work by several laboratories. This technique represents the intersection of two phenomena: 1) the ability of a cultured embryonic stem (ES) cell to remain pluripotent, 
euploid, and capable of forming germ cells in chimeric animals, and 2) the ability of a transfected piece of DNA to integrate in the ES genome by homologous recombination. The technique is owed to the combined efforts of several individuals. A great deal of the pioneering work with ES cell culture comes from Martin Evans, Elizabeth Robertson, Allan Bradley, Gail Martin, Martin Hooper, and Rolf Kemler (Evans \& Kaufman, 1981; Martin, 1981; Bradley, et al., 1984; Doetschman, et al., 1985; Hooper, et al., 1987). Much of the initial work in homologous recombination comes from the laboratories of Mario Capecchi, Oliver Smithies, and Paul Berg (Folger, et al., 1982; Smith \& Berg, 1984; Smithies, et al., 1985). In the mid 1980s, these individuals and others were able to show that by either infecting or transfecting into ES cells genes disrupted by a selectable marker one could select for a population of ES cells that contained a reciprocal exchange of an endogenous gene for the disrupted gene. This population of mutant ES cells could then be microinjected into 3.5 day old mouse blastocysts. The injected blastocysts could be surgically transferred into the uterus of a surrogate mother and the altered ES cells could develop into the various tissues of a mouse including the germ-line (Hooper, et al., 1987; Thomas \& Capecchi, 1987). Mating the resulting chimeric animals could lead to the generation of a line of mice containing the mutation (Figure 2). The technique is now recognized as one of the more powerful techniques in biology and has been applied to the study of development, immunology, neurobiology, cancer, various inborn errors of metabolism, and recently, atherosclerosis and lipoprotein metabolism.

\section{Differences in lipoprotein metabolism between mouse and human.}

Central to understanding the phenotype of transgenic and knockout mice used to study lipoprotein metabolism is a working knowledge of the differences between mouse and human lipoprotein systems. Several gross differences exist between mouse and human (Table 1). Transgenic studies have suggested a host of more subtle differences. Because of 
these differences, the mouse is not the ideal organism in which to study lipoprotein metabolism and atherosclerosis. Other small animals such as the hamster and rabbit are more suitable systems for drawing conclusions relevant to humans. However, the ability to geneticaily manipulate the mouse has led to a progressively increased use of this organism by lipoprotein biologists. We are thus in a position to accept the mouse as a model of lipoprotein physiology and atherosclerosis, dissecting apart areas in which it differs from the human, and either genetically altering these differences to create a more human-like mouse, or accounting for these differences appropriately. The latter requires careful interpretation of results and at times limited generalization of how these interpretations translate to humans.

Humans and mice significantly differ in their lipoprotein profiles. The most obvious difference between mouse and man is the lipoprotein profile. The mouse is an animal that contains $75 \%$ of its plasma cholesterol in the HDL fraction, while humans normally contain $75 \%$ of their plasma cholesterol in non-HDL fractions, and the majority in LDL (Chapman, 1986). It is unclear why mouse plasma is so enriched in HDL cholesterol as compared to VLDL and LDL cholesterol. In the fasted state, total plasma cholesterol in a typical mouse is approximately $80 \mathrm{mg} / \mathrm{dl}$, HDL cholesterol levels are $60 \mathrm{mg} / \mathrm{dl}$, VLDL cholesterol levels are $15 \mathrm{mg} / \mathrm{dl}$, and LDL levels are $5 \mathrm{mg} / \mathrm{dl}$. In humans, fasted plasma cholesterol levels vary but in an average Westerner total plasma cholesterol levels are 190 $\mathrm{mg} / \mathrm{dl}$, HDL cholesterol levels are $60 \mathrm{mg} / \mathrm{dl}$, VLDL cholesterol levels are $15 \mathrm{mg} / \mathrm{dl}$, and LDL cholesterol levels are $115 \mathrm{mg} / \mathrm{dl}$. The striking difference in LDL levels between mouse and human accounts for the major differences in lipoprotein profile.

LDL is generated from triglyceride hydrolysis of the larger VLDL and IDL particles in the circulation (reviewed in Myant, 1990). LDL contains only apoB as its protein moiety. Two forms of apoB exist, a full length apoB, termed apoB-100, and a truncated apoB of $48 \%$ 
the full-length size, termed apoB-48. ApoB- 48 is formed by the addition of a stop codon through RNA editing. In humans this editing event occurs only in the intestine (Chen, et al., 1987; Hospattanker, et al., 1987; Powell, et al., 1987). Lipoprotein particles secreted from the intestine thus contain apoB-48, while lipoprotein particles secreted from the liver, which does not possess editing potential, contain apoB-100. Only the apoB-100 containing particles can become LDL. In the mouse, however, both the liver and the intestine possess editing capability (Davidson, et al., 1988; Tennyson, et al., 1989). The mouse intestine secretes only an apoB-48-containing particle and the liver can secrete an apoB-100- and an apoB-48-containing particle. The relative decrease in apoB-100-containing particles secreted from the liver may to some extent account for the low levels of LDL in mouse plasma. This phenomenon probably does not account for the entire difference between mouse and human because approximately $50 \%$ of lipoproteins secreted from mouse liver still contain apoB-100. ApoE also is involved. ApoE is a primary ligand for the LDL receptor and the only lipoprotein ligand for the LDL receptor-related protein (reviewed in Mahley, 1988; Havel \& Kane, 1989). Mouse lipoproteins are enriched in apoE, and apoE is found in the mouse, but not in the human, in the LDL density range of 1.019 to 1.063. The presence of apoE in this density range suggests an enhanced role for apoE in mouse LDL, or LDL precursor, clearance. This enhanced role for apoE in mouse lipoprotein clearance is best illustrated in apoE-deficient mice. As described below, apoE-deficient mice develop a severe hypercholesterolemia, almost four times as severe as apoE-deficient humans.

Mice do not possess CETP. A second major difference between mouse and man that may account for differences in lipoprotein profile is the cholesterol ester transfer protein (CETP). CETP mediates the transfer of lipid between lipoprotein particles, predominantly the exchange of HDL cholesterol for VLDL triglyceride (Morton \& Zilversmit, 1983; Albers, et al., 1984; Tall, 1986). Rodents do not express CETP while humans do (Tall, 
1993). An inability to decrease HDL cholesterol and increase non-HDL cholesterol in the mouse may be related to the lack of CETP. Consistent with this postulate is the observation that humans who are deficient in CETP have elevated HDL cholesterol (Brown, et al., 1989; Inazu et al., 1990; Koizumi, et al., 1991). These individuals, however, do not have decreased non-HDL cholesterol. Also, as described below, transgenic mice that overexpress CETP have diminished HDL cholesterol, but do not have human levels of apoB-containing particles. These observations indicate that alone CETP does not account for the difference in lipoprotein profile between mouse and human. The combination of altered hepatic lipoprotein production, the potential for differences in lipoprotein clearance, and the absence of CETP, may act in conjunction to effect a diminished steady state level of murine LDL.

Other differences between human and mouse. Other differences exist between the two organisms that should be considered. The mouse lacks apo(a), a plasminogen-like plasma protein that in humans is found covalently linked to the apoB-100 of LDL by a disulfide bridge. The lipoprotein particle that contains apo(a) is termed Lp(a). High plasma levels of $\mathrm{Lp}(\mathrm{a})$ increase the risk for atherosclerosis in a dose dependent fashion (Utermann, 1989). Additionally, the mouse contains a monodisperse population of $\mathrm{HDL}$ particles (Jiao, et al., 1990). In humans, HDL particles are of three (and in some instances four) sizes (Eisenberg, 1984). In the mouse, a single HDL size fraction of approximately $10 \mathrm{~nm}$ exists (Rubin, et al., 1991a; Rubin, et al., 1991b; Chajek-Shaul, et al., 1991).

Other subtle differences exist between mouse and human that can contribute to the mouse's distinct lipoprotein profile and metabolic parameters. These differences will be considered in the discussion of the various transgenic lines. One global disparity between the two that may reflect the obvious difference in lipoprotein profile is that the mouse is an atherosclerosis resistant organism, while the human is highly susceptible to atherosclerosis 
(Stoltzfus \& Rubin, 1993). Vascular wall parameters, non-lipoprotein related plasma factors, vessel size, rheostatic forces, and life span may contribute to the murine resistance to atherosclerosis, but as demonstrated by transgenic and knockout mice, the low VLDL/LDL, high HDL lipoprotein profile and the absence of apo(a) certainly act in a significant if not primary manner to impart resistance.

\section{ApoAI}

The most well-studied apolipoprotein in transgenic mice has been apoAI, a choice target for study in transgenic mice because of its importance in determining plasma HDL levels and metabolism in humans. ApoAI is also the most abundant apolipoprotein in mouse plasma, present at over $150 \mathrm{mg} / \mathrm{dl}$. ApoAI is a $28 \mathrm{kDa}$ protein synthesized in the liver and intestine. It is found in plasma primarily in the HDL fraction. Transgenic mouse studies have been instrumental in clarifying the role of apoAI in lipoprotein physiology, understanding structural and functional differences of apoAI from different species, identifying cis-acting elements that confer its liver and intestinal specific expression, and elucidating its antiatherogenic potential.

\section{Human apoAI overexpression causes elevated mouse HDL levels. Two} laboratories have overexpressed the human apoAI gene in mice (Walsh, et al., 1989; Rubin, et al., 1991a). A range of apoAI plasma levels has been reported from barely detectable levels, to levels twice that of normal human levels. As in humans, a direct correlation exists between the plasma apoAI level in these transgenic mice and the level of HDL cholesterol. In high expressing transgenic mice HDL cholesterol levels increase to $100 \mathrm{mg} / \mathrm{dl}$ from approximately $50 \mathrm{mg} / \mathrm{dl}$ in control mice. In the latter, mouse apoAI levels are approximately $150 \mathrm{mg} / \mathrm{dl}$, while in the former total apoAI (mouse + human) levels are close to $300 \mathrm{mg} / \mathrm{dl}$. Similar increases in plasma HDL levels occur in transgenic lines created 
in hybrid or inbred genetic backgrounds. The cause of increased total plasma HDL, as indicated by kinetic studies, appears to be due to an increase in apoAI production rate . A further indication that apoAI production is critical to the establishment of plasma $\mathrm{HDL}$ levels comes from two lines of apoAI-deficient mice created by gene targeting in ES cells (Williamson et al., 1992; Plump et al., 1993). Mice lacking apoAI have HDL levels $80 \%$ lower than control mice. A range of plasma apoAI levels from zero to twice normal levels has thus been established in the transgenic and knockout mice. This range of plasma apoAI is associated with a range of $\mathrm{HDL}$ cholesterol levels from $20 \%$ normal to twice normal levels.

ApoAI overexpression causes HDL polydispersity. In addition to causing a dramatic increase in $\mathrm{HDL}$ cholesterol levels, overexpression of human apoAI also leads to an alteration of HDL particle size, composition, and kinetics (Rubin, et al., 1991a; ChajekShaul, et al., 1991). Control mouse plasma contains a monodisperse population of HDL with a mean particle diameter of about $10 \mathrm{~nm}$, similar to human HDL2b. ApoAI overexpression causes polydispersity of $\mathrm{HDL}$, generating three populations with particle sizes of $11.4 \mathrm{~nm}, 10.2 \mathrm{~nm}$, and $8.7 \mathrm{~nm}$, similar to human $\mathrm{HDL}_{1}, \mathrm{HDL}_{2}$, and HDL3. Interestingly, this trend towards a more human-like HDL profile is associated with the virtual loss of mouse apoAI from the plasma and its replacement by human apoAI. This phenomenon is apparently post transcriptional because mouse apoAI hepatic and intestinal mRNA levels are identical in control and transgenic mice. Why the mouse protein is replaced in plasma by the human protein is unknown, but the observation suggests significant structure/function differences between the two proteins. ApoAI amino acid sequence differs $30 \%$ between mouse and human (Januzzi, et al., 1992). The fact that $\mathrm{HDL}$ structure is so altered and that murine apoAI is almost non-existent in the transgenic mouse plasma suggests dominant effects of human apoAI primary amino acid sequence on HDL size. 
Human apoAI overexpression causes altered HDL metabolism. In addition to the replacement of mouse apoAI for human apoAI and the polydispersion of HDL particle size, the human apoAI transgenics also display different HDL kinetics (Chajek-shaul, et $a l ., 1991)$. HDL cholesterol ester and apoAI turnover studies indicate that HDL particles in control mice possess both a selective and a particulate route of hepatic uptake, while HDL particles in transgenic mice possess only a particulate route of uptake. The fact that replacement of human for mouse apoAI causes the loss of selective cholesterol ester uptake may reflect either the appearance of smaller HDL particles in the transgenic plasma or the ability of mouse but not human apoAI to interact with the mouse hepatocyte cell surface to stimulate selective HDL cholesterol ester uptake. Although HDL levels are increased 2-fold in high expressing transgenic lines, there is no increase in the amount of cholesterol ester that fluxes through HDL in the process of reverse cholesterol transport. This may simply reflect the fact that cholesterol ester flux is saturated in the control animals and further elevation of HDL levels has no effect on cholesterol flux. In support of this hypothesis, kinetic studies in apoAI-deficient mice demonstrate an 8-fold decrease in HDL cholesterol ester flux (Plump et al., 1993). It is unclear whether this decrease represents a decrease in tissue cholesterol ester flux or a decrease in plasma cholesterol ester flux. Regardless, these animals do not develop atherosclerosis, suggesting that a simple reduction of apoAI and $\mathrm{HDL}$ levels is not sufficient to lead to an atherosclerosis-susceptible state. This is certainly the case in humans. Although low HDL levels are correlated with markedly increased risk for atherosclerosis, not all individuals with low HDL develop clinically evident atherosclerosis (reviewed in Breslow, 1989). In both humans and mice, steady state apoAI and HDL levels do not appear to be pathognomonic of an atherosclerosis susceptible state.

\section{ApoAI overexpression leads to decreased foam cell formation in response} to an atherogenic diet. The mouse is typically an atherosclerosis resistant animal, but certain inbred strains such as the C57BL/6 inbred line will develop fatty streak lesions at 
the root of the aorta in response to a high fat, high cholesterol diet (Paigen, et al., 1985; Paigen, et al., 1990). Rubin et al. took advantage of this phenomenon and generated a line of apoAI overexpressing transgenic mice on the inbred C57BL/6 background. As compared to the control mice, the apoAI overexpressing mice were either fully or mostly protected from developing lesions (Rubin, et al., 1991b). This study represented the first clear indication that a genetic elevation of apoAI levels, and thus HDL levels, could impede the development of an atherogenic process. It has been demonstrated that several genes contribute to atherosclerosis susceptibility in $\mathrm{C} 57 \mathrm{BL} / 6$ mice, none of which map to the apoAI locus (Paigen, et al., 1987). The finding that specific elevations in the plasma level of apoAI and $\mathrm{HDL}$ can diminish atherogenesis supports a direct antiatherogenic role for these plasma constituents.

Tissue specific gene expression of the apoAI/CIII/A-IV locus. ApoAI transgenics have also been useful in understanding the regulatory elements that confer intestinal and hepatic expression of the apoAI, as well as the closely linked apoCIII gene. In humans, rats, and mice, apoAI, CIII, and A-IV exist as a cluster on the short arm of human chromosome 11 (Karathanasis, 1985; Haddad, et al., 1986; Januzzi, et al, 1992). All three are expressed solely in the intestine and liver. Initial studies in apoAI transgenic mice performed with genes containing only $256 \mathrm{bp}$ of upstream regulatory sequence demonstrated expression in the liver but not the intestine (See Figure 3a) (Walsh, et al., 1989; Rubin, et al., 1991a). This indicated the presence of an element in the apoAI proximal promoter that conferred hepatic specific expression and the absence of an intestinal enhancer element within the same stretch of DNA. Using transgenic constructions of varying lengths, Walsh et al. demonstrated that the intestinal enhancer element for the apoAI as well as the apoCII gene is contained approximately $5 \mathrm{~kb} 3^{\prime}$ to the apoAI gene, in the apoCIII promoter (Walsh, et al., 1993). The importance of these in vivo studies is underscored by tissue culture studies. A 5' and not a 3' apoAI element is necessary to 
achieve high levels of apoAI promoter expression in an intestinal cell line, $\mathrm{CACO}_{2}$ (Higuchi, et al., 1988; Sastry, et al., 1988). This same element, located between 2,052 and $41 \mathrm{bp} 5^{\prime}$ to the apoAI transcription start site does not lead to intestinal apoAI expression in transgenic mice, supporting the utility of in vivo analyses to study gene regulation. Transgenic studies should be useful in continued efforts to narrow the size of the enhancer and a targeted disruption of the element by homologous recombination in ES cells may help to unequivocally characterize the specified element as a necessary and required element for intestinal expression of the endogenous apoAI/CII/A-IV complex. The eventual discovery of the apoAI, CIII intestinal specific factor should add to our understanding the regulation of apolipoprotein gene expression in the intestine, a crucial, but poorly understood area of lipoprotein biology.

\section{ApoAII}

ApoAII is an $8.7 \mathrm{kDa}$ protein that is a major component of $\mathrm{HDL}$, comprising approximately $20 \%$ of the total HDL protein content. In humans apoAII exists as a dimer and in mice as a monomer. Transgenic studies have been critical in formulating a basic understanding of apoAII function. Transgenic studies have focused on three issues related to human apoAII: (1) an inability to affect HDL levels; (2) an effect on HDL particle size; and (3) the role of apoAII-containing HDL on cholesterol ester flux and atherosclerosis susceptibility.

\section{Effect of human apoAII overexpression on HDL levels and particle} composition. Unlike apoAI, apoAII levels do not correlate with HDL cholesterol levels in humans (Civeira, et al., 1992). Humans with apoAII deficiency have normal HDL levels and do not appear to have altered risk for the development of atherosclerosis (Deeb, et al., 1991). Recent work with transgenic mice overexpressing human apoAII has similarly shown an inability of apoAII elevations to affect $\mathrm{HDL}$ levels. Schultz et al. created 
several lines of human apoAIl overexpressing transgenic mice (Schultz, et al., 1992).

These mice had plasma apoAII concentrations ranging from 30 to $50 \mathrm{mg} / \mathrm{dl}$ (as compared to 20 - $30 \mathrm{mg} / \mathrm{dl}$ in control mice), but showed no change in HDL cholesterol levels. HDL particle structure, however, did change. In addition to the large apoAI, A-II-containing HDL particle found in the control mice, the apoAII transgenic mice had a smaller, less abundant $8.0 \mathrm{~nm}$ HDL particle that contained only apoAII. Humans with absent apoAI or with apoAI structural mutations, such as apoAISeattle (Deeb, et al., 1991), also produce an $8.0 \mathrm{~nm}$ HDL particle containing only apoAII. The human and mouse data suggest that under conditions in which human apoAII cannot interact with normal human apoAI, it has the ability to become the primary structural protein of $\mathrm{HDL}$ creating a similarly sized HDL particle in the plasma of both mice and humans. A second line of mice has been generated that contains both the human apoAI and apoAII genes (Schultz, et al., 1992). In mice overexpressing apoAI only, or apoAI and apoAII, HDL levels were similar. In both, HDL levels are elevated approximately 2 -fold. The mice differ, however, in the $\mathrm{HDL}$ protein composition. The apoAI overexpressing mice had mainly Lp(A-I) particles in their plasma, while the apoAI, A-II overexpressing mice had mainly Lp(A-I, A-II) particles in their plasma. The conclusion from the transgenic studies is similar to that of human studies: human apoAI can influence HDL particle levels and structure, while human apoAIl can only influence HDL particle structure.

\section{Effect of apoAII overexpression on cholesterol efflux and atherosclerosis} susceptibility. Several populations of HDL particles exist in humans as determined by either size or by apolipoprotein content. The majority of HDL clinical analysis is based on total HDL cholesterol or on HDL particle size. This is because epidemiological studies have suggested that the strongest indicator of atherosclerosis risk is total HDL cholesterol, and factors such as the relative composition of apoAI and apoAII may not appear to increase predictive value (Stampfer, et al., 1991). These conclusions are controversial in that 
several studies have been plagued by experimental inaccuracies in measuring apolipoprotein levels and most of these studies may not have addressed the appropriate apolipoprotein pools. Total plasma or even HDL apoAI or apoAII levels may not serve as the strongest predictors of risk. A more direct measurement of these apolipoproteins in specific $\mathrm{HDL}$ subclasses may serve as a better indicator of risk (Cheung, et al., 1991). Recent in vitro evidence suggests that the apolipoprotein content of HDL may be important in the ability of HDL to elicit cholesterol efflux from cells and thus may affect the ability of HDL to act in an anti-atherogenic manner. In humans, in terms of apolipoprotein content, two types of HDL particles predominate, those with apoAI only [Lp(A-I)], and those with apoAI and apoAII [Lp(A-I, A-II)] (Cheung \& Albers, 1982). Studies performed in tissue culture have suggested that $\mathrm{Lp}(\mathrm{A}-\mathrm{I})$ particles have a greater ability to stimulate cholesterol efflux from cholesterol loaded cells than Lp(A-I, A-II) particles (Barbaras, et al., 1987). Transgenic models have offered an excellent system in which to test these tissue culture results.

As mentioned above, lines of transgenic mice that overexpress only apoAI or apoAI and apoAII have been generated and studied. The two groups of mice differed markedly in their response to an atherogenic diet (Schultz, et al., 1993). The apoAI transgenics were protected from the development of aortic fatty streaks, as had been previously demonstrated, while the apoAI, A-II transgenics were not protected from the development of fatty streak lesions. In the two groups of mice, HDL and non-HDL cholesterol concentrations were identical, but $\mathrm{HDL}$ protein composition was different. It follows that HDL levels are not the sole determinant of atherosclerosis susceptibility in mice and that particle composition plays a significant role in determining risk. The fact that transgenic mice that overexpress human apoAI and contain $\mathrm{Lp}(\mathrm{A}-\mathrm{I})$ particles develop fewer fatty streak lesions than control mice in response to an atherogenic diet may perhaps be due to the ability of an Lp(A-I) particle to more effectively elicit cholesterol efflux from cholesterol loaded macrophages and smooth muscle cells of the artery wall. Transgenic mice that 
overexpress both human apoAI and human apoAII and contain $\mathrm{Lp}$ (A-I, A-II) particles in their plasma are significantly less protected from the development of fatty streak lesions, suggesting that $\mathrm{LP}(\mathrm{A}-\mathrm{I}, \mathrm{A}-\mathrm{II})$ particles may not possess a similar ability to elicit cholesterol efflux from cells. This reasoning assumes that reverse cholesterol transport through $H \mathrm{HDL}$ is in fact a physiological process. No direct in vivo evidence exists to support this compelling theory (reviewed in Reichl \& Miller, 1989). We cannot dismiss the possibility that the $\mathrm{Lp}(\mathrm{A}-\mathrm{I})$ particle is protective through other mechanisms such as increasing anti-oxidation potential, direct vessel wall effects, increased LCAT activation, or that the LP(A-I, A-II) particle is an atherogenic particle. These studies illustrate the utility of engineered mice in addressing the complicated mechanism of HDL's protective effects and the role of apoAI and apoAII in determining the ability of $\mathrm{HDL}$ to act in a protective fashion.

The possibility that apoAII may in fact be an atherogenic factor was raised by Warden et al. in a study in which the mouse apoAI gene was overexpressed in transgenic mice (Warden, et al., 1993). These mice were quite different than the human apoAII transgenic mice. In these studies mouse apoAIl levels were elevated approximately 3-fold as compared to control mice. Unlike the mice with the human transgene, the mice with overexpression of the mouse gene demonstrated significant differences in their lipoprotein profile. On a mouse chow diet, total cholesterol was elevated 2- to 3-fold from controls. Both HDL and non-HDL cholesterol levels increased to a similar extent in the transgenic animals. The most distinctive difference in the plasma of the mouse apoAII transgenic and either the control or human apoAII transgenic mice was the appearance of a cholesterol ester-rich particle in the LDL density range with alpha migratory ability on agarose gel electrophoresis. This particle was composed primarily of apoE and apoAII, but not apoB. Surprisingly, on a low fat mouse chow diet, the mouse apoAII transgenics developed fatty streak lesions, which were not observed in the control mice or in the human apoAII transgenic mice. The difference in lipoprotein profile and atherosclerosis susceptibility in 
the human and mouse apoAII transgenic mice may reflect species-specific differences in the apoAII gene. In addition to primary sequence differences, the mouse apoAII protein is monomeric and the human apoAII is dimeric (in human as well as in human apoAII transgenic mouse plasma). The story is also complicated by atherosclerosis susceptibility which probably does not reflect a decreased protective effect of the Lp(A-I, A-II) particle as discussed in the studies in human apoAII transgenics, but the appearance of an atherogenic particle in the plasma. This inference is suggested by the lack of fatty streak formation in the apoAI-deficient mice, which possess murine apoAII-only particles, but do not develop lesions (Plump, et al., 1993). Additionally, the mouse apoAII transgenic mice did not develop significantly different fatty streak levels in response to an atherogenic diet, suggesting that in the basal state the transgenic mice possess an atherogenic particle whose pathogenicity is unaffected by an atherogenic diet. If one assumed diminished protection in these mice than one would expect to see an enhanced effect after exposure to an atherogenic environment. Whatever the mechanism of atherosclerosis development in these animals, the message is clear that the species-specific differences in apolipoprotein primary sequences can significantly effect lipoprotein metabolism and atherogenesis.

Apo (a)

Apo (a) is a complicated apolipoprotein that is unrelated to any of the other apolipoprotein genes (Utermann, 1989). It is a plasminogen-like protein whose size is determined by the number of Kringle domains. An inverse correlation has been established within populations, between apo(a) levels and apo(a) size. Apo (a) is found in human plasma in a covalently linked complex with apoB-100 and the lipoprotein particle with which apo(a) is associated, known as Lp(a), is similar to LDL with the addition of the apo(a) protein Elevations in apo(a) and $L p(a)$ have been strongly correlated with increased risk for the development of atherosclerosis (Utermann, 1989). The genetics of apo(a) have proved to 
be quite complex, with both transcriptional and post-transcriptional mechanisms, as well as the rate of plasma catabolism determining apo(a) plasma levels. The genetic complexity of the apo(a) and the fact that primates are the sole experimental system in which apo(a) physiology can currently be studied make the establishment of engineered animal models critical. Our ability to understand how $L p(a)$ levels are regulated and how $L p(a)$ levels may be therapeutically reduced depends on these models. The mouse does not possess apo(a), but a line of apo(a) transgenic mice has been generated.

\section{Apo (a) overexpression in mice leads to primarily free apo(a) in plasma.} Chiesa et al. created a line of apo(a) transgenics in a hybrid C57BL/6 $\mathrm{x}$ SJ mouse in order to assess both the atherogenic potential of the protein as well as its metabolic parameters (Chiesa, et al., 1992). Apo (a) plasma levels of $10 \mathrm{mg} / \mathrm{dl}$ were generated in these mice, levels similar to median levels in humans. In these mice apo(a) was secreted into plasma but the majority of the protein was found in a lipid-free fraction with only $5 \%$ associating with lipid. The cause of low lipid binding was demonstrated to be structural differences between mouse and human apoB and not the low levels of LDL normally present in mouse plasma. Reinfusion of human but not mouse LDL into the transgenic mouse plasma led to a rapid covalent association of apo(a) with the lipid fraction. This observation confirmed that apo(a) could associate with LDL in a post-hepatic process and that although we cannot rule out that in humans apo(a) and LDL associate intracellularly, free apo(a) does possess the ability to associate with LDL in plasma.

\section{The presence of apo(a) in plasma increases fatty streak formation in} response to an atherogenic diet. When fed an atherogenic, cholesterol-rich diet for 3.5 months, the apo(a) transgenic mice developed nascent fatty streak lesions while control mice did not develop substantial lesions (Lawn, et al., 1992). This result is striking given the observation that the majority of apo(a) was free in plasma. Several mechanisms have 
been described for how $L p(a)$ might act to increase atherosclerosis risk. These include the preferential uptake of the $\mathrm{Lp}$ (a) particle by macrophages, the ability of apo(a) to attract lipid to the vessel wall by first binding to sites such as fibrin or fibronectin, the inhibition of thrombolytic activity, the ability of bound apo(a) to act as a chemotactic factor for monocytes, or the ability of apo(a) to induce smooth muscle cell proliferation. Although we cannot dismiss the possibility in the transgenics that the 5\% of apo(a) in the lipid-bound fraction is the atherogenic component of the plasma, the atherosclerosis in the apo(a) transgenic mice strongly suggests that free apo(a) can be atherogenic. This would likely support the hypothesis that apo(a) first binds to components of the vessel wall and secondarily acts to exert its atherogenic potential. Uncertainty exists as to whether its major affect is the recruitment of lipid, the recruitment of cells, the initiation or propagation of smooth muscle proliferation, or the inhibition of fibrinolysis. Apo (a) was found to localize in the fatty streaks by immunocytochemistry. Co-localized with the apo(a) was apoB. These two observations suggest that either the lipid bound fraction was atherogenic or apo(a) was able to direct apoB-containing lipoproteins to the vessel wall.

To address the question of apo(a) pathogenicity a line of human apoB transgenic mice will be necessary. Recently mice expressing human apoB and human apo(a) have been created (Callow, et al., 1994). Human like L $\mathrm{L}$ (a) particles are efficiently assembled with the human apo(a) nearly completely associated with apoB via a disulfide bond in the plasma of these doubly transgenic mice. One can now compare atherosclerosis susceptibility in apo(a) only, apo(a)/human apoB, and human apoB only transgenic mice.

The future of apo (a) transgenics. Though intriguing in terms of the connection between apo (a) and atherosclerosis, the preceeding studies are marred by two deficiencies that make the understanding of $\mathrm{Lp}$ (a) metabolism and apo (a) gene regulation difficult. The first is due to the lack of significant apo (a) association with mouse apo B. Because the 
majority of apo (a) is free in plasma, the current line of transgenic mice will serve as a poor model for understanding $L p(a)$. The recently developed animals making $L p(a)$ should overcome this deficiency (Callow, et al., 1994). A second problem arises from the construction used to generate the apo (a) transgenic mice. The transgene was an apo (a) cDNA driven by an heterologous promoter. The use of a heterologous promoter will preclude studies that might address apo (a) gene regulation. To fully understand the determinants of apo (a) plasma levels we must understand both the plasma processes involved in metabolism and the trans and cis elements controlling gene expression. Further studies using the apo (a) promoter driving either reporter genes or the apo (a) gene should be key in elucidating regulatory elements in the apo (a) gene as well as in understanding processes that regulate apo (a) protein trafficking within the cell.

\section{Apo B}

ApoB is an ideal gene to study in transgenics, in part because of its importance in determining the metabolic parameters of lipoproteins in humans, and in part because the mouse apoB gene differs funcrionally than the human gene. At least two significant differences exist between the human and mouse apoB genes: (1) mouse apoB is edited to the truncated apoB-48 in the liver, and (2) mouse apoB is unable to bind apo(a). Both parameters, discussed in detail in other sections of this chapter, may account for some of the marked differences between the mouse and human lipoprotein systems. Expression of a full-length human apoB gene in the mouse should allow a more in depth understanding of VLDL and LDL metabolism. In addition, this same system will allow the study of $L p(a)$ metabolism as well as its genetics, which has proven particularly complicated.

Expression of human apoB in mice results in an animal with a human-like lipoprotein profile. Unfortunately, because of its size (the human apoB cDNA is 
approximately $14 \mathrm{~kb}$ and the gene is over $40 \mathrm{~kb}$ ), several laboratories have had difficulty in stably expressing the gene in mice. Recently, however, human apoB transgenics have been created by microinjection of a genomic apoB clone contained on a phagemid vector (Callow, et al., 1994). These mice efficiently edit human apoB in the liver. High level expression of the human apoB transgene resulted in human-like LDL and HDL concentrations in animals fed a low fat, low cholesterol diet. These animals have overcome one of the major deficiencies of the mouse as a model for human lipoprotein metabolism, that being low LDL. The fact that major lipoprotein of the apoB transgenic animals is now LDL and not HDL markedly improves this organism as a model to study issues relevant to human lipoprotein metabolism. When the apoB transgenics are crossed with apo(a) transgenics, efficient assembly of $\mathrm{Lp}(\mathrm{a})$ takes place in the plasma. Nearly all the apo(a) in these animals is now found associated through a disulfide bond to human apoB containing LDL particles. This line of animals should prove instrumental in understanding the metabolism of $\mathrm{Lp}(\mathrm{a})$ and apoB-containing particles.

\section{Replacement of the mouse $\mathrm{apoB}$ gene for a truncated gene results in a} syndrome similar to hypobetalipoproteinemia. Homanics et al. have recently been able to replace the endogenous full-length apoB gene with a truncated gene that encodes an apoB protein of $70 \%$ the size, terméd apoB-70 (Homanics, et al., 1993). In humans, several mutations that cause truncations in the apoB gene have been found to associate with the disease familial hypobetalipoproteinemia (reviewed in Farese, et al., 1992). The apoB modification used in this transgenic study was designed to create a situation in the mouse that mimicked familial hypobetalipoproteinemia, a disease that results in decreased plasma apoB, cholesterol, and B-migrating lipoproteins. The apoB-70 replacement mice manifested several abnormalities remarkably similar to those seen in humans with familial hypobetalipoproteinemia. Mice homozygous for the alteration had reductions in plasma total cholesterol, HDL-cholesterol, and triglyceride, as well as a fasting chylomicronemia, 
and B-migrating lipoproteins--findings typically seen in humans with the disease. Humans with hypobetalipoproteinemia are typically resistant to the development of atherosclerosis. Studies aimed at addressing the susceptibility of the apoB-70 mice to atherosclerosis should prove informative. In addition to the abnormalities similar to those seen in humans, the apoB-70 mice also developed hydrocephalus. $32 \%$ of mice homozygous for apoB-70 were found to develop hydrocephalus, a condition not seen in any of the control mice. The cause of this unusual phenotype is not certain and has been documented in only one line of mice, and may be the result of a non apoB related abnormality of the ES line or may reflect an abnormality in absorption. Humans with hypobetalipoproteinemia develop problems associated with fat malabsorption and have difficulty absorbing fat soluble vitamins. These individuals, if untreated, often develop sequelae of vitamin E deficiency. Maternal vitamin E deficiency in other laboratory animals has also led to hydrocephalus in offspring. The apoB-70 mice appear to have reduced plasma levels of alpha-tocopherol, which may help to explain the predisposition to this unusual pathology.

\section{ApoCs}

Transgenic mouse technology has been instrumental in defining an in vivo role for the apoC genes in triglyceride metabolism. The apoCs are a group of three apolipoprotein genes, apoCI, CII, and CIII, that were named as a group not because of homology, but because of similarity in size as seen initially by SDS-PAGE of HDL apolipoproteins. Studies have established that apoCII is a cofactor in the lipoprotein lipase (LPL) mediated lipolysis of VLDL triglyceride, and humans who are apoCII-deficient have type I hyperlipoproteinemia, a disease characterized by fasting hypertriglyceridemia (Cox, et al., 1978; Santamarina-Fojo, 1992). There exists a great leniency in this system, as individuals can have as little as $10 \%$ normal apoCII levels and still be normolipidemic. Aside from the extreme situation of apoCII deficiency there is little clinical evidence that apoCII 
overexpression, or either apoCI or apoCIII under or overexpression can affect lipoprotein metabolism. In vitro evidence from the early 1980s, however, suggested that each of the apoCs were capable of inhibiting lipolysis of triglyceride rich particles (Windler, et al., 1980; Windler \& Havel, 1985). ApoCI and apoCII were also shown to be capable of inhibiting the association of apoE with the LDL receptor and the possible remnant receptor, the LDL receptor-like protein (LRP) (Weisgraber, et al., 1990). Transgenic studies have furthered these in vitro observations, demonstrating that each of the apoCs can cause hypertriglyceridemia when overexpressed. The transgenic data has proved invaluable in understanding potential functions of the apoCs, and investigators are now in the process of examining the role of apoCs in triglyceride metabolism in humans.

\section{ApoCIII overexpression causes a dose-dependent hypertriglyceridemia.} ApoCIII was the first of the apoCs to be studied in transgenic mice. Ito $e t$ al. overexpressed the human apoCIII gene and found that transgenic animals were severely hypertriglyceridemic (Ito, et al., 1990). ApoCIII caused hypertriglyceridemia in a dosedependent fashion. In the fasted state low expressing apoCIII transgenic mice developed a 2-fold elevation in plasma triglyceride and high expressing mice a 10 -fold elevation. Mechanistic studies in the apoCIII transgenic mice demonstrated a delay in particle clearance as a possible cause of the hypertriglyceridemia. One must also consider an abnormality in lipolysis to explain the exaggerated hypertriglyceridemia in the face of only mild hypercholesterolemia. Aalto-Setala et al. showed, however, that there was no difference in the ability of transgenic VLDL to act as a substrate for soluble LPL and that there was no change in tissue LPL activity in the transgenic mice (Aalto-Setala, et al., 1992). Though informative, these analyses do not consider the ability of a triglyceride-rich particle to associate with LPL bound to heparan sulfate in the basement membrane. Recent work has suggested that interactions between lipoproteins and either their cognate receptor or LPL requires not only a direct association between the particle and the receptor or lipase 
but also an interaction between components of the particle and the basement membrane (Eisenberg, et al., 1992; Ji, et al., 1993). These studies suggest that domains in apoE are capable of interacting in a positive fashion with heparin sulfate in the basement membrane and that the apoCs, because of their low isoelectric points, inhibit association. These observations might apply to the apoCIII transgenic mice, because in addition to an increase in apoCIII per VLDL particle, there is a decrease in apoE. The observed delay in particle clearance might thus reflect the lipolytic defect, and not be the primary cause of hypertriglyceridemia. Furthermore, the experiments assessing the activity of soluble LPL did not consider basement membrane effects, potentially masking any lipolytic defects. Regardless of the mechanism, it will be interesting to determine if humans manifest a similar response to apoCIII overexpression.

\section{ApoCI and apoCII overexpression also cause hypertriglyceridemia. Studies} have suggested that the apoCII and apoCI genes can have similar effects on triglyceride metabolism. Shachter et al. demonstrated that in a similar fashion to apoCII, apoCII overexpression caused a fasting hypertriglyceridemia with plasma triglyceride levels proportional to plasma apoCII levels (Shachter, et al., 1993). This study similarly showed a delay in VLDL catabolism as well as an increased ratio of apoC:E. Simonet $e t$ al. have presented a line of transgenic mice that overexpressed the apoCI gene (Simonet, et al., 1991). High levels of apoCI in these mice also led to hypertriglyceridemia. The apoCI analysis did not include any characterization of the lipoprotein profile or any possible mechanism of action, but from in vitro analysis we may assume that if this effect is real, its action is probably similar to that of apoCII and apoCIII. The mechanism of delayed VLDL triglyceride metabolism in the various apoC transgenic mice remains to be established, but the fact that apoC overexpression in mice can cause hypertriglyceridemia suggests a possible etiology of primary hypertriglyceridemia in humans, an hypothesis that has been formulated to a large extent from transgenic studies. 


\section{ApoE}

Apolipoprotein $\mathrm{E}$ is a $34 \mathrm{kDa}$ glycoprotein found in mouse plasma in association with lipoproteins of all densities. Like other apolipoprotein genes it is expressed predominantly in the liver, unlike other apolipoprotein genes low levels of expression occur in most other tissues. ApoE is involved in the receptor mediated clearance of chylomicron remnant and IDL particles from the circulation, as well as in several other processes related to lipoprotein and cellular cholesterol metabolism. These include involvement in reverse cholesterol transport, in the local redistribution of cholesterol between cells, and in macrophage cholesterol homeostasis (reviewed in Mahley, 1988). ApoE is complex in terms of its effects on lipoprotein metabolism, its tissue specific gene regulation, and its ability to determine disease state. Transgenic and knockout mice have been instrumental in dissecting the role that apoE plays in each of these areas. Overexpression of apoE has demonstrated that there are several tissue specific cis-acting elements upstream and downstream of the apoE gene. Overexpression and targeted disruption of the apoE gene has been helpful in understanding the physiology of apoE as well as its role in atherogenesis.

\section{Identification of a liver specific enhancer located $15 \mathrm{~kb} 3$ ' to the apoE}

gene. Two laboratories have been actively pursuing through transgenic technology an enhancer element that confers hepatic specific expression to the apoE gene. The story emerged as a complicated one when Simonet $e t a l$. and Smith $e t$ al. were able to demonstrate, using a similar transgene that contained approximately $5.0 \mathrm{~kb}$ of $5^{\prime}$ human apoE flanking sequence and $2.0 \mathrm{~kb}$ of 3 ' flanking sequence, that the majority of transgene expression occurred in the kidney with little liver expression (see Figure 3b) (Simonet, et al., 1990a; Smith, et al., 1990). Control mice typically express high levels of apoE in the 
liver and low levels in the kidney. This result suggested the existence of a kidney suppressor and a liver enhancer element that was not contained within this basic construction. Using a variety of DNA fragments containing the apoE gene and a variable amount of 5 and $3^{\prime}$ flanking sequence, Simonet et al. demonstrated that by including $23 \mathrm{~kb}$ of downstream sequence, one could achieve high levels of liver expression and basal levels of kidney expression (Simonet, et al., 1990b). Shachter et al. made use of a tissue culture system to refine this $23 \mathrm{~kb}$ fragment to a minimal 154 base pair element that was capable of directing apoE liver specific expression in transgenic mice (Shachter, et al., 1993). This element appears capable of imparting hepatic specific expression to two other apolipoprotein genes clustered with the apoE gene, the apoCI and apoCII genes. Perhaps in a fashion similar to the locus control region of the beta globin gene cluster (reviewed in Epner, at al., 1991), this liver cluster can mediate liver specific expression of a group of related genes. A targeted disruption of this element in ES cells may be necessary to unequivocally demonstrate that this element is in fact required for expression of all three genes. Transgenic studies have thus elucidated a concept of coordinated expression that now appears to hold for two apolipoprotein gene clusters, the apoE/CI/CII and the apoAI/CII/A-IV loci. These are examples of experiments that are difficult or impossible to perform in other systems.

ApoE overexpression diminishes VLDL and LDL levels. Using heterologous promoters to direct high levels of rat apoE expression, Shimano et al. demonstrated that apoE overexpression led to a decrease in VLDL and LDL cholesterol (Shimano, et al., 1992). This decrease in non-HDL cholesterol levels was due primarily to an increase in the rate of clearance of apoB-containing particles. In addition to this decrease in non-HDL cholesterol on a low fat chow diet, apoE overexpression was found to diminish increases in plasma cholesterol after a high fat, high cholesterol diet. These studies required high apoE plasma levels, levels 5 -fold greater than the average human and mouse, but suggest that 
apoE possesses the ability to significantly accelerate the removal of apoB-containing particles from the blood and to effect a change in steady state particle levels. Similar results have been obtained in LDL receptor overexpressing transgenic mice (see below), demonstrating that overexpression of either ligand or receptor can result in a similar phenotype.

\section{ApoE deficiency causes severe hypercholesterolemia due to B-VLDL} accumulation. Using homologous recombination in ES cells, Zhang et al. and Plump et al. were able to specifically disrupt the apoE gene and generate apoE-deficient mice (Zhang, et al., 1992; Plump, et al., 1992). On a mouse chow diet, apoE-deficient mice were found to have a significant accumulation of apoB-containing particles in the blood, with total plasma cholesterol levels increasing almost 10 -fold from $60 \mathrm{mg} / \mathrm{dl}$ in control mice to almost $500 \mathrm{mg} / \mathrm{dl}$ in apoE-deficient mice. This increase in total plasma cholesterol was due to an increase in VLDL, DL, and LDL-cholesterol, with the greatest increase occurring in the VLDL fraction. Kinetic studies suggested that the cause of this elevated VLDL-cholesterol was delayed particle clearance from the blood. The VLDL from these mice were found to be cholesterol ester enriched B-VLDL similar to the abnormal particles found in humans with type III hyperlipoproteinemia or apoE deficiency. Similar to apoE-deficient humans, the apoE-deficient mice did not manifest a significant hypertriglyceridemia. In addition to the exaggerated hypercholesterolemia on a low fat diet, the apoE-deficient mice were found to be sensitive to dietary intervention, manifesting a 4-fold elevation in VLDL and total plasma cholesterol in response to a high fat Western-type diet. This extreme abnormality in the apoE-deficient mice (plasma cholesterol level of approximately $2,000 \mathrm{mg} / \mathrm{dl}$ on a Western-type diet) was much more severe than the corresponding abnormality in apoEdeficient humans who typically develop plasma cholesterol levels of $500 \mathrm{mg} / \mathrm{dl}$ on Westerntype diets (Ghiselli, et al., 1981; Schaefer, et al., 1986; Mabuchi, et al., 1989; Kurosaka, et al., 1991; Lohse, et al., 1991). The cause of this discrepancy reflects species differences 
between humans and mice, including an increase in the amount of apoB-48 in mouse and the greater role of apoE in lipoprotein clearance in mice.

\section{The apoE-deficient mice develop extensive, fibroproliferative}

atherosclerosis. The extreme lipoprotein abnormality in the apoE-deficient mice led to the development of severe atherosclerosis (see Figure 4). Unlike lesions in the dietinduced atherosclerosis prone mice and in other genetically manipulated mice, the quality of lesions in the apoE-deficient was more severe in terms of progression and distribution (Zhang, et al., 1992; Plump, et al., 1992; Nakashima, et al., 1994). Lesions in this animal are observed on a chow diet and are exaggerated by the Western diet. Under both dietary conditions, monocyte adhesion occurs at least as early as 6 weeks of age, fatty streak lesions appear by eight to 10 weeks of age, and fibroproliferative lesions can occur as early as 15 weeks of age. The lesions distribute throughout the entire aorta with a predilection for forming in the root of the aorta, the ascending thoracic aorta, the lesser curvature of the aortic arch, and the abdominal aorta at the ostia of major vasculature such as the celiac, superior mesenteric, and renal arteries, as well as at the aortic bifurcation. In addition, the mouse develops lesions in other major vessels such as the brachiocephalic, subclavians, carotids and pulmonary arteries. In terms of both distribution and composition, the lesions in this mouse appear to mimic lesions seen in humans. This animal is promising as a small animal model of atherosclerosis and should provide valuable insight into understanding the pathogenesis of atherosclerotic lesions as well as the genetic, environmental, and therapeutic approaches to treating this deadly disease. The apoE-deficient mouse has advantages over the existing mouse models of atherosclerosis which require the feeding of toxic diets for extended periods of time in order to induce nascent lesions. As an example of the utility of the apoE-deficient mouse as a model of atherosclerosis, one might be able to assess the ability of genes such as apoAI or CETP to modify both lesion initiation and lesion progression in a rapid fashion. One could assess effects on fatty streak formation in 
animals as young as 10 weeks and effects on fibroproliferative changes in animals as young as 15 weeks. In addition one could assess the ability of modifying factors to affect atherosclerosis in a purely genetic fashion without dietary intervention.

\section{Overexpression of trans dominant apoE mutants in mice leads to a} syndrome similar to type III hyperlipoproteinemia. Two additional apoE transgenic models provide further insight into the involvement of apoE in determining atherosclerosis susceptibility. Maagdenberg et al. and Fazio et al. have created lines of transgenic mice that overexpress dominant mutant apoE genes found in individuals with type III hyperlipoproteinemia who are not homozygous for the apoE2 allele (Maagdenberg, et al., 1993; Fazio, et al., 1993). The apoE3-Leiden allele, described in a large Dutch family with a high incidence of type III hyperlipoproteinemia, contains a tandem duplication of amino acids 120-126 of the apoE gene. The apoE3142 allele contains a missense mutation with a cys for arg replacement at codon 142 . When overexpressed in mice both mutant genes led to a type III hyperlipoproteinemic syndrome similar to that seen in humans with an abnormality in plasma cholesterol and triglyceride metabolism. In a dose-dependent manner both sets of transgenic mice demonstrated hypertriglyceridemia and hypercholesterolemia, and experiments performed in the apoE3142 mutant suggest delayed particle clearance. It is intriguing to note that the levels of mutant protein in the plasma of these mice was much greater than the corresponding levels in humans. Also, the appearance of B-VLDL, a particle typically found in the plasma of type III hyperlipoproteinemic individuals, occurred only after a threshold plasma level of mutant protein was reached. Both groups of mice developed atherosclerotic lesions on a mouse chow diet, with exaggerated and dispersed lesions forming in response to an atherogenic, high cholesterol diet. In both cases lesion formation was not as severe as that seen in the apoE-deficient mice. This may be a reflection of the presence of mutant or wild-type apoE or of a concomitant hypertriglyceridemia in the trans-dominant transgenics. 


\section{CETP}

CETP is a plasma protein that catalyzes the transfer of lipid among lipoprotein particles. CETP is most effective in exchanging cholesterol ester in HDL for triglyceride in VLDL (Morton \& Zilversmit, 1983; Albers, et al., 1984; Tall, 1986). Because the activity of CETP leads to changes in kinetic parameters of lipid and lipoprotein particles, as well as changes in steady state levels, it has been a difficult protein to fully understand. In addition, there has been contention over the years as to whether CETP is an atherogenic protein because it tends to decrease steady-state plasma HDL levels, or an anti-atherogenic protein because it may act to augment reverse cholesterol transport by increasing HDL cholesterol ester flux to the liver. Animal models have proved valuable to the overall understanding of CETP function and atherogenic potential. A range of CETP actives has been described in many organisms from absent in rodents to moderate levels in humans to high levels in rabbits and monkeys (Ha \& Barter, 1982). In general the amount of CETP activity in plasma has been indirectly correlated with the amount of $\mathrm{HDL}$ and directly correlated with an animal's predisposition to develop atherosclerosis. (The pig is a noted exception where CETP levels are low or absent, but atherosclerosis susceptibility high). Additionally, a population of CETP-deficient humans has been described in Japan (Brown, et al., 1989; Inazu, et al., 1990; Koizumi, et al., 1991). These individuals have HDL-cholesterol levels between $200 \mathrm{mg} / \mathrm{dl}$ and $300 \mathrm{mg} / \mathrm{dl}$, but no data exists to suggest whether these individuals are at a decreased risk of developing atherosclerosis. Mice are similarly a CETP-deficient species with high HDL:total cholesterol ratios and resistance to atherosclerosis. Transgenic studies involving CETP overexpression in the mouse have offered a great deal to the understanding of CETP function and its atherogenic potential. 


\section{CETP can decrease mouse HDL levels in a process that is accelerated by}

human apoAI. Agellon et al. first reported that overexpression of CETP in transgenic mice resulted in a mild decrease in HDL cholesterol (Agellon, 1991). Hayek et al. later showed that this decrease in HDL cholesterol was exaggerated when transgenic mice overexpressing CETP were crossed with transgenic mice overexpressing the human apoAI gene (Hayek, et al., 1992). In the absence of apoAI CETP elicited a 35\% decrease in HDL cholesterol and in the presence of apoAI, CETP was able to elicit a $66 \%$ decrease in HDL cholesterol. In the CETP only transgenics, $\mathrm{HDL}$ particles were large and monodisperse; in the human apoAI, CETP transgenics HDL particles were polydisperse, similar to those of humans apoAI only transgenics. The intriguing difference between the CETP only and the human apoAI, CETP mice was that $100 \%$ of plasma CETP associated with $\mathrm{HDL}$ in the human apoAI, CETP mice while only $22 \%$ of CETP associated with HDL in the CETP only mice. In the former, the majority of CETP associated with the smaller HDL particles. This striking observation suggests an interaction between the human apoAI gene and CETP. The predilection of CETP association with small HDL in the apoAI, CETP transgenics indicates that CETP may not necessarily interact directly with apoAI, but may possess a higher affinity for small $\mathrm{HDL}$ particles. In addition to its increased association with $\mathrm{HDL}, \mathrm{CETP}$ was able to increase the $\mathrm{HDL}$ cholesterol ester fractional catabolic rate (FCR), presumably by catalyzing the transfer of $\mathrm{HDL}$ cholesterol ester into apoBcontaining particles. This increased HDL cholesterol ester FCR was probably responsible for the decrease in $\mathrm{HDL}$ in these mice, but for unknown reasons, did not lead to an increase in the amount of cholesterol ester in apoB-containing particles.

A second intriguing study that demonstrated a gene interaction was shown by Hayek et al. in a line of mice overexpressing three human genes: CETP, apoAI, and apoCIII (Hayek, et al., 1993). A long-standing relationship has been established in humans between hypertriglyceridemia and low HDL cholesterol. Several lines of evidence suggest that a 
hypertriglyceridemic state promotes exchange of HDL cholesterol ester for VLDL triglyceride and results in a diminution of $\mathrm{HDL}$ levels. To establish a hypertriglyceridemic environment in the presence of high CETP activity, the apoCIII gene was overexpressed in the presence of CETP and human apoAI. Although the hypertriglyceridemia generated by apoCIII overexpression caused a modest decrease in HDL levels, substantial decreases in HDL cholesterol occurred only in the presence of the CETP gene, an effect exaggerated by the additional presence of the apoAI gene. The cause for decreased $\mathrm{HDL}$ in these triple transgenic mice was primarily an increase in $\mathrm{HDL}$ cholesterol ester FCR. The conclusion from these analyses is that CETP has the ability to significantly affect HDL cholesterol levels and metabolism, and in a hypertriglyceridemic state CETP can further reduce HDL cholesterol presumably by augmenting the exchange of VLDL triglyceride for $\mathrm{HDL}$ cholesterol ester. Though hypertriglyceridemia can elicit a modest reduction in HDL cholesterol levels, it appears that CETP has a significant role in determining the high triglyceride-low HDL cholesterol relationship.

A third transgenic study has demonstrated that not only are plasma triglycerides important in understanding CETP function, but plasma cholesterol is as well. Studies have previously shown that dietary or genetic insults that result in elevated plasma cholesterol levels also result in elevated plasma CETP and CETP activity (Quinet, et al., 1990; McPherson, et al., 1991; Martin, et al., 1993). Jiang et al. have shown, by overexpressing a CETP mini-gene with its natural flanking promoter, that this is a transcriptional effect (Jiang, et al., 1992). In response to a high fat, high cholesterol diet, these mice had a 2.5 - fold increase in plasma CETP activity and an associated 4-fold increase in hepatic CETP mRNA. This effect was not seen in transgenic mice overexpressing a CETP mini-gene driven by a heterologous metallothianein promoter. 
CETP has an atherogenic effect on the response of C57BL/6 mice to an atherogenic diet. Given the tendency of CETP to diminish HDL cholesterol levels in the transgenic mice, a question of interest is whether the gene is capable of acting in an atherogenic manner. Alternatively, one might expect CETP to act in an anti-atherogenic manner by increasing HDL cholesterol ester flux and stimulating the movement of cholesterol from tissues to the liver. Marotti et al. addressed the atherogenic potential of CETP by overexpressing the monkey CETP gene in mice (Marotti, et al., 1992). This study made use of transgenic mice expressing high CETP activity. The monkey gene is 2.5-fold more active than the human gene and the transgenic mice used in this study had CETP levels 5-fold higher than average monkey levels; thus, CETP activity was greater than 15 -fold over average human levels, as compared to approximately 2 -fold over human levels in the previous studies. The lipoprotein profile in the simian CETP transgenics was slightly different than the lower expressing human CETP transgenics. The monkey gene caused a similar 50\% reduction in HDL cholesterol on both a chow and atherogenic diet, as well as a $50 \%$ to $75 \%$ increase in VLDL + LDL cholesterol on both diets, a phenomenon not observed in the human transgenics. The high expressing CETP mice developed a greater degree of fatty streak lesions in the proximal aorta than control mice. In addition, there was a trend, though not statistically significant, in lesion development between control, low, and high expressing mice. Of greatest interest to this study was that a very strong direct correlation was observed between the (VLDL + LDL):HDL ratio and the degree of lesion development (Marotti, et al., 1993). By eliciting higher atherogenic potential and lower anti-atherogenic potential in response to the diet, CETP was able to cause increased lesion formation.

\section{Lipoprotein Receptors}


The LDL receptor is the only lipoprotein receptor so far studied in mice. An attempt was made to knockout the LRP, but a homozygous deletion of the LRP resulted in a developmental lethal phenotype, probably related to non-lipoprotein processes (Herz et al., 1992). Unfortunately, this has precluded experiments addressing whether the LRP is the remnant receptor and, if so, to what extent the receptor functions in lipoprotein clearance.

Mice that overexpress or underexpress the LDL receptor have proved more informative. In addition to demonstrating an effect on steady state and diet-induced LDL levels (Hofmann, et al., 1988; Yokode, et al., 1990), LDL receptor overexpressing mice have also demonstrated that $\mathrm{Lp}$ (a) can be recognized by the LDL receptor in vivo (Hofmann, et al., 1990) and that a carboxy terminal signal sequence is necessary to direct proper basolateral localization of the receptor (Yokode, et al., 1992). Mice that lack the LDL receptor have also provided a workable model in which one can rapidly study LDL receptor gene therapy, a treatment currently in human clinical trials (reviewed in Grossman \& Wilson, 1992).

\section{LDL receptor overexpression decreases LDL in chow fed transgenic mice} and prevents LDL increases in transgenic mice fed a high fat diet. The LDL receptor was one of the first genes involved in lipoprotein metabolism to be overexpressed in mice. Hoffman et al. reported in 1988 that overexpression of an LDL receptor gene driven by a metallothianein promoter led to high levels of LDL receptor activity in the liver when mice were challenged with cadmium sulfate (Hofmann, et al., 1988). This initial study demonstrated marked increases in hepatic lipoprotein uptake and the virtual elimination of apoB and apoE from the plasma. Because of toxicity associated with chronic administration of cadmium salts this transgene precluded studies aimed at addressing the effect of prolonged high levels of LDL receptor activity in an animal challenged with an atherogenic diet. In order to study the effect of prolonged diet in LDL receptor overexpressing animals, a second LDL receptor transgene was constructed under the 
control of the mouse transferin promoter (Yokode, et al., 1990). As expected, high levels of LDL receptor overexpression was able to prevent a rise in LDL levels following prolonged administration of a high fat, high cholesterol diet. In these studies, FPLC lipoprotein profiles demonstrated that the high fat, high cholesterol diet increased plasma VLDL, IDL, and LDL levels in control animals, while the transgenic animals developed only a slight increase in VLDL levels, with no increase in $\mathrm{DL}$ or LDL cholesterol. In the control mice there was an expected increase in plasma apoE and apoB in response to the diet, while levels of these same proteins did not change in the transgenic animals. It follows from these results that under certain circumstances the level of LDL receptor activity in the liver can be rate-limiting to the clearance of circulating LDL. Unfortunately, no studies have emerged suggesting that these same animals are able to resist fatty streak formation in the proximal aorta.

\section{LDL receptor deficient mice have elevated LDL levels and delayed clearance} of VLDL and LDL particles. Ishibashi et al. have generated a line of LDL receptor deficient mice by gene targeting in ES cells (Ishibashi, et al., 1993). Both heterozygous and homozygous deficient mice developed elevated total plasma cholesterol levels. Kinetic studies demonstrated a significant delay in both VLDL and LDL clearance from the plasma of the LDL receptor knockout mice as compared to control mice. It is interesting to note that humans with LDL receptor deficiency have more severe elevations in LDL levels than these knockout mice. Humans with familial hypercholesterolemia often develop total plasma cholesterol levels of $1,000 \mathrm{mg} / \mathrm{dl}$, while the homozygous deficient mice developed total plasma cholesterol levels of $250 \mathrm{mg} / \mathrm{dl}$. This might reflect an enhanced role of apoE in lipoprotein clearance in mice, an hypothesis further supported by the exaggerated hypercholesterolemia apoE-deficient mice. As mentioned previously, apoE deficiency in mice causes a more severe hypercholesterolemia than apoE deficiency in humans. When 
fed an equivalent diet apoE-deficient mice develop plasma cholesterol levels of 2,000 $\mathrm{mg} / \mathrm{dl}$, levels 4-fold higher than apoE-deficient humans.

LDL receptor-deficient mice provide a model for gene therapy. In addition to providing insight into lipoprotein metabolism, the LDL receptor-deficient mice have provided a model in which to experiment with gene therapy. In the Ishibashi et al. study, a replication defective adenoviral vector was used to replace LDL receptor in the knockout mice. High levels of LDL receptor expression generated in these mice reversed the lipoprotein defect and led to a baseline lipoprotein profile. The power of the adenoviral system is exceptional and not unique to these LDL receptor deficient mice. Similar experiments have been performed in Watanabee rabbits (Wilson, et al., 1990; Chowdhury, et al., 1991; Wilson, et al., 1992) and human clinical trials are under way with retroviral vectors. The advantage of the LDL receptor-deficient mice is that extensive studies can be performed with large numbers of animals in a relatively rapid fashion. These studies will need to address problems associated with adenovirus mediated gene therapy such as immune response and long term expression of the therapeutic genes.

\section{Summary and Future Directions}

Evidenced by the large number of overexpressing and gene knockout animals and the amount of information derived from these models, transgenic technology has become an invaluable tool to lipoprotein biologists. Genetic manipulation has vastly improved our understanding of $\mathrm{HDL}$ and triglyceride metabolism. It has confirmed existing hypotheses surrounding the metabolism of LDL and it has offered insights into the role of one particularly elusive protein, CETP. Both gene knockout and transgene overexpression have offered avenues by which one can alter the lipoprotein system to generate mice that are at increased risk for the development of atherosclerosis. The studies described in this chapter 
have helped shape new concepts as well as refine existing ones. Perhaps of greatest excitement is that there is more to come. There are a host of lipoprotein related genes as well as human mutant forms of these genes yet to be overexpressed, and most of the endogenous murine analogues have yet to be knocked out. In addition to completing the repertoire of genes, the future should bring studies that make use of a primary asset of the mouse, genetics. Through the combination of inter-transgenic crosses, classical mouse genetics, and an emerging genome map, investigators should be able to identify known and anonymous genes that can suppress or enhance many of the exaggerated phenotypes described above. For example, are there loci in the mouse genome that can increase or decrease the susceptibility to atherosclerosis seen in the apoE-deficient, apo(a), CETP, apoAII, and apoE trans-dominant transgenic mice? An emerging field of DNA technology in which large pieces of DNA carried in phagemids or on YACs should prove useful to lipoprotein biology in the elucidation of cis-acting elements that confer tissue and regulatable expression patterns on certain genes, as well as in overexpressing some of the larger genes that have so far proven elusive. The establishment of regulatable promoters that can be turned on or off should allow for further studies not possible in existing systems. The generation of models in which to test gene therapy is certainly another potential power of these engineered animals. The LDL receptor-deficient mouse has already provided one successful environment in which to explore gene therapy possibilities.

In addition to the scientific possibilities provided by transgenic technology, there is a second emerging and critical area, finances. The cost involved in maintaining laboratory animals is large. Though mice are less expensive than many of the larger animals, the large numbers of lines and genetic crosses established at many institutions often offsets the savings generated by individual mice. Techniques such as cryopreservation of mouse embryos should provide an alternative to the expensive maintenance of whole lines. In addition, the ready distribution of animals among laboratories through a central supplier or 
by good will should help ease the burden of each laboratory having to maintain several different lines. The Jackson Laboratory (Bar Harbor, Maine) has already begun to make some of the more informative engineered animals available at a reasonable cost. As a final consideration of transgenic technology that certainly has financial implications, is the establishment of transgenic technology in other species. As discussed at the beginning of this chapter, differences between the lipoprotein system in the mouse and in man and the resistance of the mouse to atherosclerosis, suggest that mice are not the ideal organism in which to study lipoprotein metabolism. Though expensive, efforts are underway establishing transgenic and gene knockout technology in species whose lipoprotein profiles more closely resemble humans, such as the rabbit and pig. 
Transgenics.chapter/Rubin, Plump

File: Tables/Figures.transgenics

Text font: Times (12 pt.)

Figures and Tables

Table 1. Differences in lipoprotein physiology between humans and mice,

Human

High LDL, low HDL

Low LDL or binding affinity of human apoB

Only hepatic apoB editing

Lesser relative role for apo $E$ in plasma lipoprotein metabolism

CETP activity present

Apo (a) and Lp(a) present

Polydisperse HDL

Atherosclerosis sensitive
Mouse

Low LDL, high HDL

High LDL or binding affinity of mouse apoB

Hepatic and intestinal apoB editing

Greater relative role for apo $E$ in plasma lipoprotein metabolism

CETP activity absent

Apo (a) and Lp(a) absent

Monodisperse $\mathrm{HDL}$

Atherosclerosis resistant 
Fig. 1 The creation of transgenic mice from embryo microinjection. 1) Male and female mice are placed together. 2) 12 hours following mating, fertilized embryos containing two pronuclei (one sperm and one ovum derived) are isolated. 3) The fertilized embryo is held by a holding pipette and injected with cloned DNA fragments. 4) Injected embryos are reimplanted into the oviducts of a hormonally-receptive female. 5) 21 days following implantation pups are born. 6) DNA is isolated from the tales of the pups and screened for the presence of the injected sequences by Southern blot analysis. 
Figure 2. Use of embryonic stem cells to create gene-targeted mice. (1) Murine embryonic stem (ES) cells are cultured in vitro (in this example, ES cells are derived from a black mouse). (2) ES cells are transfected with DNA of the chosen cloned gene that contains the desired mutation (depicted by the $\mathrm{X}$ ). The mutation is transferred to the genome of the ES cell by homologous recombination. (3) A small percentage of the ES cells contain the modified endogenous gene (those cells depicted with an X). (4) Targeted ES cell clones are isolated and expanded using positive and/or negative selection schemes. (5) The targeted ES cells are microinjected into blastocyst-stage embryos (in this example, the blastocyst is derived from a white mouse). (6) The injected blastocyst is transferred into a recipient female. (7) A chimeric animal is born. The chimeric mouse exhibits coat color mosaicism due to the difference in coat color alleles of the ES cells and the recipient blastocyst. Germ-line transmission of the targeted gene is determined by breeding the chimeric mice. (8) Heterozygous siblings are bred together to produce animals that are homozygous for the desired mutation. 
Figure 3. Tissue specific transcriptional elements in the apo (A) A-I/ CIII/A-IV and (B) apo E/CI/CII gene loci. Shown are the two apolipoprotein gene clusters with genes indicated by the solid boxes and intergenic sequences indicated by thin lines. Some common restriction sites are listed. Shown below each of the clusters are some of the transgenes and their tissue specific expression patterns. L, Liver; I, Intestine; $K$, Kidney; S, Skin; B, Brain. (We thank Dr. Jonathan Smith and Dr. Annemarie Walsh, Rockefeller University, for providing the information.) 
Figure 4. Time course of the lesions of atherosclerosis in apo E-deficient mice fed a low fat chow or high fat Western-type diet. As shown, apo Edeficient mice develop progressive atherosclerotic lesions. The progression is accelerated by a Western-type diet. (Figure from Nakashima, et al., 1994) 